\title{
Mixed Traffic Equilibrium Assignment Model Based on Reliability of Travel Time
}

\author{
Liu Yansong \\ Shandong Management University, Shandong250100 \\ Jinan75623446@qq.com
}

\begin{abstract}
The objective of transportation network equilibrium analysis is to know about the distribution of traffic flow on network, for ensuring network state and providing theoretical reference for improvement design of transportation network. The paper analyzes route choice behavior based on travel time reliability under uncertainty, and establishes road choice model based on travel time reliability. The paper proposes several travel time definitions. And the road choice model under uncertainty is applied to equilibrium distribution of transportation network to analyze the distribution of network flow of travel time reliability under different situations. And the paper analyzes the reality that several traffic modes exist on network, and takes accounts of the situations that traffic modes influence each other and don't influence each other on network. The paper analyzes route choice behavior of traveler under information condition. Combined with the factors of mixed traffic and reliability route choice behavior, the paper researches the traffic equilibrium partitioning models under two distributing criterions.
\end{abstract}

Keywords: reliability, network equilibrium, travel time, multi-type users, multimode

The rapid and sustainable development of social economy makes urbanization and motorization in our country develop rapidly, which makes the traffic demand increase sharply. In order to the increasing traffic demand, the investment on transportation infrastructure construction is great and the speed is rapid, which achieves development. However, there are more and more traffic problems. The traffic is heavy, the order is confused, the accidents happen frequently, and the pollution is aggravated. The urban transport problems have become the bottleneck problem to limit the development of social economy [1].

The transportation network reliability is the concentrated expression of transportation network system performance. The research indicates that reliable road network not only can reduce the going-out cost of the user, but also can increase the benefit level of the society. In intelligent transportation system, reliable network system is a necessary condition to implement ITS. Studying route choice behavior of the user under uncertainty and the interaction between the transportation system and traveler under the condition of information is one of the key points of ITS. Reliability research can provide effective judgment criteria and theoretical basis for ITS, which makes ITS improve the operation efficiency of transportation network system effectively.

The real traffic network is a mixed network including many transportation modes (such as bus, car and non-motor vehicle) and many routing criterions. The diversity of modes makes the equilibrium of traffic flow on network complicated and difficult to analyze. The difference of mastering traffic information makes the traffic information mastered by different users on network different. How to analyze the network equilibrium under the condition of mixed transportation is very important. The paper observes the transportation equilibrium under the condition of many transportation modes and many information criterions, and considers them by combining them. And the paper establishes a combined 
equilibrium assignment model to analyze the equilibrium of transportation network under the mixed condition.

\section{Multi-mode Mixed Traffic Network Equilibrium Assignment}

\subsection{Interdependent Mixed Traffic Assignment between Modes}

The transportation modes on traffic network don't influence each other or the traffic flow is interdependent. The travel time delay of each transportation mode on network only depends on the flow of the traffic mode. Like the mixed transportation network consisting of the car, bus, subway and non-motor vehicle, the travel time delay of them on network only depends on the transportation network mode (under the ideal condition).

Which transportation mode is selected by the traveler depends on the utility of the traveler for the mode. The utility includes the reliability factors of selecting the transportation mode. If the transportation mode to be selected by the traveler only relates to the travel time reliability and average travel time of the mode, according to utility maximization theory, the probability of selecting the $i$ transportation is

$$
P_{w}^{i}=P\left(T^{w, i} \leq T^{w, l}, \forall l \in K, l \neq i\right)
$$

In the formula, ${ }^{i}{ }^{i}$ means the probability of $\mathrm{OD}$ on selecting the $i$ transportation between $w, \hat{T}^{w, i}$ means the optimal and reliable travel time of OD on selecting the $i$ transportation between $w, T^{w, i}=\pi_{w}^{i}+\xi_{w}^{i} . \pi_{w}^{i}$ means the minimal optimal reliable travel time of OD on the I mode between . $\xi_{w}^{i}$ means the error of recognizing optimal reliable travel time on the i transportation mode.

If the error of the traveler recognizing the optimal and minimal reliable travel time on $i$ transportation mode obeys Gumbel distribution, the selection model based on Logit can be deducted as follows.

$$
P_{w}^{i}=\frac{\exp \left(-\gamma \pi_{w}^{i}\right)}{\sum_{i \in K} \exp \left(-\gamma \pi_{w}^{i}\right)}
$$

In the formula, $\gamma$ means the randomness of the cognitive error on the optimal reliable travel time of the i mode.

If the traveler on network can accurately realize the network state and travel time impedance, the equilibrium condition of multi-mode determinate user assignment according to determinate user balance principle.

$$
\left.\begin{array}{l}
q_{w}^{i}=p_{w}^{i} q_{w} \\
f_{k}^{w, i}-\pi_{w}^{i} \geq 0 \\
f_{k}^{w, i}\left(f_{k}^{w, i}-\pi_{w}^{i}\right)=0
\end{array}\right\}
$$

In the formula, $q_{w}^{i}$ means the traffic volume of OD on the i traffic mode between w, $f_{k}^{w, i}$ means the traffic volume of OD on the $\mathrm{i}$ traffic mode between $\mathrm{w}$ on route $\mathrm{k}$, and $\pi^{i}{ }^{i}$ means the minimal optimal reliable travel time of OD on the $\mathrm{i}$ traffic mode between $\mathrm{w}$.

As there are many transportation modes on network, the following equivalent mathematical programming models can be written as follows.

$$
\min Z\left(x_{a}^{1}, x_{a}^{2}, \ldots \ldots x_{a}^{i}, q_{w}^{1}, q_{w}^{2}, \ldots \ldots q_{w}^{i}\right)=\sum_{i} \sum_{a} \int_{0}^{x_{a}} c_{a}^{i}(\omega) d \omega+\frac{1}{\gamma} \sum_{w} \sum_{i}\left(q_{w}^{i} \ln q_{w}^{i}-q_{w}^{i}\right)
$$




$$
\begin{gathered}
\text { s.t. } \sum_{k} f_{k}^{w, l}=q_{w}^{l} \quad l=1,2, i . \\
q_{w}=\sum_{l=1}^{i} q_{w}^{l} \\
f_{k}^{w, l} \geq 0 \quad l=1,2, i . \\
q_{w}^{l} \geq 0 \quad l=1,2, \ldots i \\
\sum_{w \in W} \sum_{k \in p_{w}} f_{k}^{w, l} \delta_{a, k}^{w, l}=x_{a}^{l} \quad l=1,2, i .
\end{gathered}
$$

$x_{a}^{i}$ means the traffic volume of the i traffic mode on the road section a, $q_{w}^{i}$ means the traffic demand of OD on the $\mathrm{i}$ traffic mode between $\mathrm{w}, f_{k}^{w, i}$ is the traffic volume of OD on the $\mathrm{i}$ traffic mode of the road $\mathrm{k}$ between $\mathrm{w}$, and $\delta_{a, k}^{w, l}$ is a switch variable.

The equivalence proves the generalized Lagrange function constructing the mathematical model.

$$
\begin{gathered}
L=\sum_{i} \sum_{a} \int_{0}^{x_{a}^{i}} c_{a}^{i}(\omega) d \omega+\frac{1}{\gamma} \sum_{w} \sum_{i}\left(q_{w}^{i} \ln q_{w}^{i}-q_{w}^{i}\right)+\sum_{i} \pi_{w}^{i}\left(q_{w}^{i}-\sum_{k} f_{k}^{w, i}\right)+\sum_{i} \pi\left(q_{w}-\sum_{i} q_{w}^{i}\right) \\
-\sum_{i} \mu_{i} q_{w}^{i}-\sum_{i} \lambda_{i} f_{k}^{w, i}
\end{gathered}
$$

$\pi^{i}{ }^{i}$ is the Lagrange multiplier corresponding to constraint (4b), $\pi_{\text {is lagrangian }}$ function corresponding to traffic-flow conservation constraint (4c), and $\lambda_{i}$ and $\mu_{i}$ are Lagrange multipliers corresponding to nonnegative constraint $(4 \mathrm{~d})$ and $(4 \mathrm{e})$. According to K-T condition, Lagrangian functions need to satisfy the following conditions at the extreme value point.

$$
\begin{gathered}
\frac{\partial L}{\partial q_{w}^{i}}=0, \quad \mu_{i} q_{w}^{i}=0, \quad \mu_{i} \geq 0 \\
\frac{\partial L}{\partial f_{k}^{w, i}}=0, \quad \lambda_{i} f_{k}^{w, i}=0, \quad \lambda_{i} \geq 0
\end{gathered}
$$

As

$$
\frac{\partial L}{\partial q_{w}^{i}}=\frac{1}{\gamma} \ln q_{w}^{i}+\pi_{w}^{i}-\pi-\mu_{i}=0
$$

When $q_{w}^{i}>0, \mu_{i}=0$, we can get from formula (6) that

$$
q_{w}^{i}=\exp \left[-\gamma\left(\pi_{w}^{i}-\pi\right)\right]
$$

From flow conservation constraint (4b), we can get

$$
q_{w}=\sum_{i} \exp \left[-\gamma\left(\pi_{w}^{i}-\pi\right)\right]
$$

And then we can get

$$
p_{w}^{i}=\frac{\exp \left[-\gamma\left(\pi_{w}^{i}-\pi\right)\right]}{\sum_{l} \exp \left[-\gamma\left(\pi_{w}^{l}-\pi\right)\right]}=\frac{\exp \left[-\gamma \pi_{w}^{i}\right]}{\sum_{l} \exp \left[-\gamma \pi_{w}^{l}\right]}
$$

$\mu_{w}^{i}$ is the minimal and optimal reliable travel time of OD on w, and formula (9) is the equilibrium condition of mode selection. 
As

$$
\frac{\partial L}{\partial f_{k}^{w, i}}=c_{k}^{w, i}-\pi_{w}^{i}-\lambda_{i}=0
$$

And when $f_{k}^{w, i}>0, \lambda_{i}=0$, we can get $c_{k}^{w, i}=\pi_{w}^{i}$. When $f_{k}^{w, i}=0, c_{k}^{w, i}>\pi_{w}^{i}$, it is the user equilibrium condition. Overall, the model at extreme value point meets the equilibrium condition, and the model is an equivalent model.

The network state and travel time impedance of the travel on network is a cognitive error. If the interdependence of errors obeys Gumbel distribution, the route selection model can be achieved. If these errors obey normal distribution, all error joint probability density functions are multivariate normal distribution functions, which can get route selection model of Probit. Route selection model of Probit has many advantages, but it has great calculated amount compared with Logit. If the route selection model of Logit is used, the equilibrium condition of multi-mode random user assignment can be achieved according to stochastic user equilibrium principle.

$$
\left.\begin{array}{l}
q_{w}^{i}=p_{w}^{i} q_{w} \\
f_{k}^{w, i}=p_{w, k}^{i} q_{w}^{i} \\
f_{k}^{w, i} \geq 0
\end{array}\right\}
$$

In the formula, $P_{w}^{i}=\frac{\exp \left(-\gamma \pi_{w}^{i}\right)}{\sum_{l \in K} \exp \left(-\gamma \pi_{w}^{l}\right)}$, and $\gamma$ means the randomness of minimum efficient reliable travel time cognitive error of each transportation mode. $p_{k}^{w, i}=\frac{\exp \left(-\theta_{i} c_{k}^{w, i}\right)}{\sum_{l \in p_{w}} \exp \left(-\theta_{i} c_{l}^{w, i}\right)},{ }^{\theta_{i}}$ means the randomness of the route reliable travel time cognitive error of the $\mathrm{i}$ mode on network. The equilibrium assignment model of multi-mode traffic network based on logit route selection under random condition can be expressed by the model in formula (12).

$$
\begin{gathered}
\min Z\left(x_{a}^{1}, x_{a}^{2} \ldots x_{a}^{i}, f_{k}^{w, 1}, f_{k}^{w, 2}, \ldots \ldots f_{k}^{w, i}, q_{w}^{1}, q_{w}^{2}, \ldots \ldots . q_{w}^{i}\right)=\sum_{w} \sum_{i} \sum_{k} \int_{0}^{x_{a}^{i}} c_{a}^{i}(\omega) d \omega+\frac{1}{\theta} \sum_{w} \sum_{i}\left(f_{k}^{w, i} \ln f_{k}^{w, i}-f_{k}^{w, i}\right) \\
+\frac{1}{\gamma} \sum_{w} \sum_{i}\left(q_{w}^{i} \ln q_{w}^{i}-q_{w}^{i}\right) \\
\text { s.t. } \sum_{k} f_{k}^{w, l}=q_{w}^{l} \quad l=1,2, i \\
q_{w}=\sum_{l=1}^{i} q_{w}^{l} \\
f_{k}^{w, l} \geq 0 \quad l=1,2, i \\
q_{w}^{l} \geq 0 \quad l=1,2, \ldots i
\end{gathered}
$$

In the formula, $x_{a}^{i}$ means the flow of the $\mathrm{i}$ transportation mode on road section a, $q_{w}^{i}$ is the traffic flow of OD on the $\mathrm{i}$ transportation mode between w. $f_{k}^{w, i}$ is the traffic flow of OD on the i transportation mode on route k between $\mathrm{w} . \delta_{a, k}^{w, l}$ is a switching variable. The model (12) is a model which is equivalent to equilibrium condition (11). 


\subsection{Interactive Mixed Traffic Assignment between Modes}

The above chapter observes the equilibrium assignment that transportation modes have no influence mutually. In fact, the hypothesis in the above chapter is ideal. The transportation modes are interactive on network. The model in the above chapter is suitable for the situation that transportation modes have no influence mutually such as subway and routine bus system. The chapter ignores the hypothesis that transportation modes are interdependent, and supposes that the transportation modes are interactive. The transportation network equilibrium assignment under interactive condition is observed.

There are many transportation modes on network and the transportation modes are interactive, $c_{a}^{i}=c_{a}^{i}\left(x_{a}^{1}, x_{a}^{2}, \ldots \ldots x_{a}^{i}\right)$ can be used to represent the reliable travel time function. It has the following characteristics according to real traffic condition [5]:

Under the non-free flow state, the average travel time increases with the increase of traffic flow,

$$
\frac{\partial c_{a}^{i}\left(x_{a}^{1}, x_{a}^{2}, \ldots \ldots x_{a}^{i}\right)}{\partial x_{a}^{i}}>0
$$

The resistive influence of each transportation mode (margin impedance) exceeds the marginal influence of other modes on the load.

$$
\frac{\partial c_{a}^{i}\left(x_{a}^{1}, x_{a}^{2}, \ldots \ldots x_{a}^{i}\right)}{\partial x_{a}^{i}}>\frac{\partial c_{a}^{i}\left(x_{a}^{1}, x_{a}^{2}, \ldots \ldots x_{a}^{i}\right)}{\partial x_{a}^{j}} \quad i \neq j
$$

There are two kinds of relationship between marginal contribution of bus load to private cars and the marginal contribution of private car load to bus impedance.

$$
\frac{\partial c_{a}^{i}\left(x_{a}^{1}, x_{a}^{2}, \ldots x_{a}^{i} \ldots x_{a}^{n}\right)}{\partial x_{a}^{j}}=\frac{\partial c_{a}^{j}\left(x_{a}^{1}, x_{a}^{2}, \ldots x_{a}^{j} \ldots x_{a}^{n}\right)}{\partial x_{a}^{i}}
$$

Or

$$
\frac{\partial c_{a}^{i}\left(x_{a}^{1} x_{a}{ }_{a}, x^{i} \cdot{ }_{a} x .{ }^{j}\right)_{a}}{\partial x_{a}^{j}} \neq \frac{\partial c\left({ }_{a}^{j}{ }_{a}{ }^{1} x_{a}{ }^{2}, \underset{a}{x},{ }^{j} x_{a_{a}}\right)}{\partial x_{a}^{i}}
$$

We name the transportation equilibrium assignment meeting the formula (15) and formula (16) as symmetric and asymmetric mixed equilibrium assignment of marginal resistance. Under symmetric type, we can establish an equivalent mathematic programming model to represent equilibrium assignment under mixing condition. The equivalent mathematical programming model is $\min Z\left(x_{a}^{1}, x_{a}^{2}, \ldots \ldots x_{a}^{n}\right)=$

$$
\begin{aligned}
& \frac{1}{n} \sum_{a}\left\{\int_{0}^{x_{a}^{1}}\left[c_{a}^{1}\left(\omega, x_{a}^{2}, x_{a}^{3}, \ldots, x_{a}^{n}\right)+c_{a}^{1}\left(\omega, 0, x_{a}^{3}, \ldots, x_{a}^{n}\right)+\ldots+c_{a}^{1}\left(\omega, . . x_{a}^{i-1}, 0, x_{a}^{i+1} \ldots x_{a}^{n}\right)+\ldots+c_{a}^{1}\left(\omega, x_{a}^{2}, \ldots x_{a}^{n-1}, 0\right)\right]\right. \\
& +\int_{0}^{x_{a}^{2}}\left[c_{a}^{2}\left(x_{a}^{1}, \omega, x_{a}^{3}, \ldots, x_{a}^{n}\right)+c_{a}^{2}\left(0, \omega, x_{a}^{3}, \ldots, x_{a}^{n}\right)+\ldots+c_{a}^{2}\left(x_{a}^{1}, \omega, . . x_{a}^{i-1}, 0, x_{a}^{i+1} \ldots x_{a}^{n}\right)+\ldots+c_{a}^{2}\left(x_{a}^{1}, \omega, \ldots x_{a}^{n-1}, 0\right)\right] \\
& \left.\quad+\ldots+\int_{0}^{x_{a}^{n}}\left[c_{a}^{n}\left(x_{a}^{1}, x_{a}^{2}, x_{a}^{3}, \ldots, \omega\right)+c_{a}^{n}\left(0, x_{a}^{2}, x_{a}^{3}, \ldots, \omega\right)+\ldots+c_{a}^{n}\left(x_{a}^{1}, x_{a}^{2}, \ldots x_{a}^{i-1}, 0, x_{a}^{i+1} \ldots \omega\right)+\ldots . .+c_{a}^{n}\left(x_{a}^{1}, x_{a}^{2}, \ldots 0, \omega\right)\right]\right\} \quad(17 \mathrm{a}) \\
& \quad \text {.t. }\left\{\begin{array}{l}
\sum_{k} f_{k}^{w, i}=q_{w}^{i} \\
f_{k}^{w, i} \geq 0 \\
\sum_{w \in W} \sum_{k \in p_{w}} f_{k}^{w, i} \delta_{a, k}^{w, i}=x_{a}^{i}
\end{array}\right.
\end{aligned}
$$


In the formula, $x_{a}^{i}$ means the traffic flow of the $\mathrm{i}$ transportation mode on route section a, $c_{a}^{i}$ means the travel time impedance of the $\mathrm{i}$ transportation mode on route section a. The model is an equivalent model. Due to space limitations, the proof procedure is not introduced.

When mixed transportation operates under action mechanism described in formula (16), the Jacobian matrix corresponding impedance function is asymmetric. It is similar to equilibrium assignment of Smith. M. J, the equivalent extremum model can't be established, which makes its optimal solution is mixed UE solution. But diagonalization algorithm can solve the problem. The diagonalization algorithm process is [6].

The algorithm is based on solving a series of standard UE sub-problems, which means that a sub-problem needs to be solved. If the link flow on the $\mathrm{n}$ iteration is ${ }^{X_{A}(n)}$, the $\mathrm{UE}$ sub-problem to be solved is

$$
\begin{aligned}
\min \tilde{Z}^{n}(X)=\sum_{a} \sum_{i=1}^{n} \int_{0}^{x_{a}^{i}} c_{a}^{i(n)}\left(x_{a}^{1(n)}, x_{a}^{2(n)}, \ldots x_{a}^{i-1(n)}, \omega, x_{a}^{i+1(n)}, \ldots, x_{a}^{j(n)}\right) d \omega \\
\text { s.t. }\left\{\begin{array}{l}
\sum_{k} f_{k}^{w, i}=q_{w}^{i} \\
f_{k}^{w, i} \geq 0 \\
\sum_{w \in W} \sum_{k \in p_{w}} f_{k}^{w, i} \delta_{a, k}^{w, i}=x_{a}^{i}
\end{array}\right.
\end{aligned}
$$

In objective function, $c_{a}^{i}$ is the function of $x_{a}^{i}$, and the flow on other route sections is flexible. $\tilde{c}_{a}^{i}\left(x_{a}^{i}\right)$ is the impedance function of route section a of the $\mathrm{n}$ iteration when the other flows are flexible,

$$
\tilde{c}_{a}^{i}\left(x_{a}^{i}\right)=c_{a}^{i}\left(x_{a}^{1(n)}, x_{a}^{2(n)}, \ldots x_{a}^{i-1(n)}, \omega, x_{a}^{i+1(n)}, \ldots, x_{a}^{j(n)}\right) 。
$$

The procedure of the algorithm is:

Step0: initialization. The initial feasible flow is $X^{n}$ and $n=0$.

Step1: solving diagonalization algorithm (18) can use any standard UE algorithm, and the produced flow is $X^{n+1}$.

Step2: convergence check. If $X^{n} \approx X^{n+1}$, it stops, or $\mathrm{n}=\mathrm{n}+1$, and it turns to the first step.

The common convergence check is that checking the following formula.

$$
\frac{1}{A} \sum_{a} \frac{\left|x_{a}^{n+1}-x_{a}^{n}\right|}{x_{a}^{n+1}} \leq \varepsilon
$$

$\mathrm{A}$ is the number of route sections on network, and $\mathrm{c}$ is a small arithmetic number.

\section{Two-criterion Traffic Equilibrium Assignment Model based on Traffic Information}

\subsection{Two-criterion Traffic Equilibrium Assignment Model under Informational Influence}

If the total traffic flow $q_{w}$ of OD on w has been given,

$$
q_{w}=\tilde{q}_{w}+\hat{q}_{w} \quad w \in W
$$

In the formula, $\tilde{q}_{w}$ means the traffic flow of the traveler with vehicle infrastructure of OD on w, and $\hat{q}_{w}$ means the traffic flow of the traveler without vehicle infrastructure of 
OD on w. The network equilibrium assignment model based on travel time reliability under informational condition can be represented by the following mathematical programming model.

$$
\begin{aligned}
& \min Z(\tilde{f}, \hat{f}, x)=\sum_{w} \sum_{a} \int_{0}^{x_{0}} c_{a}(\omega) d \omega+\frac{1}{\theta} \sum_{w \in W} \sum_{k \in p_{w}} \hat{f}_{k}^{w} \ln \hat{f}_{k}^{w} \\
& \text { s.t. } \sum_{k \in p_{w}} \tilde{f}_{k}^{w}=\tilde{q}_{w} \quad w \in W \\
& \sum_{k \in p_{w}} \hat{f}_{k}^{w}=\hat{q}_{w} \quad k \in p_{w} \quad w \in W \\
& \tilde{f}_{k}^{w} \geq 0 \quad k \in p_{w} \quad w \in W \\
& \hat{f}_{k}^{w} \geq 0 \quad k \in p_{w} \quad w \in W \\
& x_{a}=\sum_{w \in W} \sum_{k \in P_{w}}\left(\hat{f}_{k}^{w}+\tilde{f}_{k}^{w}\right) \delta_{a, k}^{w}
\end{aligned}
$$

The formula (21b) and (21c) are the flow conservation constraints of the travelers with vehicle infrastructure and without vehicle infrastructure. And the formula (21d) and formula (21e) are non-negativity constraints of flow.

The equivalence proof,

The formula (21b) is a minimum problem with linear equality constraint and non-negativity constraint. To prove that the mathematical programming model is equivalent to mixed equilibrium condition, the generalized Lagrangian function of the mathematical programming model needs to be firstly constructed, as follows.

$$
L=Z+\sum_{w} \lambda_{w}^{1}\left(\tilde{q}_{w}-\sum_{k} \tilde{f}_{k}^{w}\right)-\sum_{w} \sum_{k} \tilde{f}_{k}^{w} \tilde{\mu}+\sum_{w} \lambda_{w}^{2}\left(\hat{q}_{w}-\sum_{k} \hat{f}_{k}^{w}\right)-\sum_{w} \sum_{k} \hat{f}_{k}^{w} \hat{\mu}
$$

In the formula, $\lambda_{w}^{1}$ and $\lambda_{w}^{2}$ are lagrangian functions of flow conservation constraint (21b) and (21c). $\tilde{\mu}_{\text {and }} \hat{\mu}$ are lagrangian functions of flow non-negativity constraints equality (21d) and (21e).

According to Kuhn-Tucker condition, the lagrangian functions must meet the following conditions on extreme point.

$$
\begin{array}{lll}
\partial L / \partial \tilde{f}_{k}^{w}=0 & , \quad \tilde{f}_{k}^{w} \tilde{\mu}=0, & \tilde{\mu} \geq 0 \\
\partial L / \partial \hat{f}_{k}^{w}=0, & \hat{f}_{k}^{w} \hat{\mu}=0, \quad \hat{\mu} \geq 0
\end{array}
$$

For the traveler with ATIS,

$$
\partial L / \partial \tilde{f}_{k}^{w}=c_{k}^{w}-\lambda_{w}^{1}-\tilde{\mu}=0
$$

As $\tilde{f}_{k}^{w} \tilde{\mu}=0, \tilde{f}_{k}^{w}>0, \tilde{\mu}=0$. We can see from formula (23) that $c_{k}^{w}-\lambda_{w}^{1}=0$, that is, $c_{k}^{w}=\lambda_{w}^{1}$. When $\tilde{f}_{k}^{w}=0$, as $\tilde{\mu} \geq 0, c_{k}^{w}=\lambda_{w}^{1}+\tilde{\mu} \geq \lambda_{w}^{1}$., for the traveler with ATIS, the route traffic flow meets the determinate equilibrium condition, and the lagrangian function is the minimal optimal reliable travel time of OD on $\mathrm{w}$.

For the traveler withoute ATIS,

$$
\partial L / \partial \hat{f}_{k}^{w}=c_{k}^{w}+\frac{1}{\theta}\left(\ln \hat{f}_{k}^{w}+1\right)-\lambda_{w}^{2}-\hat{\mu}=0
$$

If $\hat{f}_{k}^{w}=0$, there is no $\partial L / \partial \hat{f}_{k}^{w}$, and formula (23) is successful when $\hat{f}_{k}^{w}>0$. From the formula $\hat{f}_{k}^{w} \hat{\mu}=0$, we can see that $\hat{\mu}=0$. And the above formula can be rewritten as

$$
\partial L / \partial \hat{f}_{k}^{w}=c_{k}^{w}+\frac{1}{\theta}\left(\ln \hat{f}_{k}^{w}+1\right)-\hat{\mu}=0
$$


According to formula (26), the route traffic flow of the traveler without ATIS is

$$
\hat{f}_{k}^{w}=\exp \left[-\theta\left(c_{k}^{w}-\lambda_{w}^{2}\right)-1\right]
$$

From the flow conservation condition (21c), we can see that

$$
\hat{p}_{k}^{w}=\frac{\exp \left[-\theta c_{k}^{w}\right]}{\sum_{l} \exp \left[-\theta c_{l}^{w}\right]}
$$

It is the randomly balanced equilibrium condition.

In fact, if the traffic information provided by the information system of the traveler is not real, the traveler with vehicle infrastructures can't select route according to UE principle. But for the traveler without vehicle infrastructures, the travelers with vehicle infrastructures can receive the information provided by the information system of traveler, the cognitive error on route travel time will reduce greatly, so the model can be applied to common situations.

$$
\begin{aligned}
& \min Z(\tilde{f}, \hat{f}, x)=\sum_{w} \sum_{a} \int_{0}^{x_{a}} c_{a}(\omega) d \omega+\frac{1}{\tilde{\theta}} \sum_{w \in W} \sum_{k \in p_{w}} \tilde{f}_{k}^{w} \ln \tilde{f}_{k}^{w}+\frac{1}{\hat{\theta}} \sum_{w \in W} \sum_{k \in p_{w}} \hat{f}_{k}^{w} \ln \hat{f}_{k}^{w} \\
& \text { s.t. } \sum_{k \in p_{w}} \tilde{f}_{k}^{w}=\tilde{q}_{w} \quad w \in W \\
& \sum_{k \in p_{w}} \hat{f}_{k}^{w}=\hat{q}_{w} \quad r \in p_{w} \quad w \in W \\
& \tilde{f}_{k}^{w} \geq 0 \quad r \in p_{w} \quad w \in W \\
& \hat{f}_{k}^{w} \geq 0 \quad r \in p_{w} \quad w \in W \\
& x_{a}=\sum_{w \in W} \sum_{k \in P_{w}}\left(\hat{f}_{k}^{w}+\tilde{f}_{k}^{w}\right) \delta_{a, k}^{w}
\end{aligned}
$$

Similarly, the model (200) can be proved to be an equivalent model. And the proof procedure is not introduced.

\subsection{Model Solution Algorithm and Examples}

1) Model solution algorithm

The following algorithm is used to solve the formula (21). According to the market share of ATIS setting, the travelers are divided, and the total amount $q_{w}$ of OD is divided into $\tilde{q}_{w}$ and $\hat{q}_{w}$.The algorithm procedure is

Step0: determining effective route set, and applying improved Dial algorithm.

Step1: according to $c_{a}(0)$, the effective route impedance can be computed. And the route flow $\tilde{f}_{k(1)}^{w}$ of the traveler with ATIS is computed. The formula (27) is used to compute the route flow $\hat{f}_{k(1)}^{w}$ of the traveler without ATIS, $\mathrm{n}=1$.

Step2: $\quad f_{k(n)}^{w}\left(f_{k(n)}^{w}=\tilde{f}_{k(n)}^{w}+\hat{f}_{k(n)}^{w}\right)$ is used to compute new route impedance. The route flow $\tilde{h}_{k(n)}^{w}$ of the traveler with ATIS is computed by using all-or-none algorithm. And the formula (27) is used to compute route flow $\hat{h}_{k(n)}^{w}$ of the traveler without ATIS.

Step3: Computing step size. 
$3.10 \leq \tilde{\alpha} \leq 1$, and $\tilde{f}_{k(n+1)}^{w}=(1-\alpha) \tilde{f}_{k(n)}^{w}+\alpha \tilde{h}_{k(n)}^{w}$. The following problems are solved to determine step size, $\tilde{\alpha}=\tilde{\alpha}^{*}$

$$
\min Z(\tilde{\alpha})=\sum_{a} \int_{0}^{x_{a}^{n+1}} c_{a}(\omega) d \omega+\frac{1}{\theta} \sum_{w \in W} \sum_{k \in p_{w}} \hat{h}_{k(n)}^{w} \ln \hat{h}_{k(n)}^{w} \text {, in which } \quad x_{w} \sum_{k} f_{k(n+1)} \delta_{a, k}^{w} \text {, and }
$$

the traffic flow of the user with ATIS on updating route is $\tilde{f}_{k(n+1)}^{w}=(1-\alpha) \tilde{f}_{k(n)}^{w}+\alpha \tilde{h}_{k(n)}^{w}$ 、

$3.20 \leq \hat{\alpha} \leq 1$, and $\hat{f}_{k(n+1)}^{w}=(1-\alpha) \hat{f}_{k(n)}^{w}+\alpha \hat{h}_{k(n)}^{w}$ is used to solve a series searching problems to determine step size, $\hat{\alpha}=\alpha^{*}$

$$
\min Z(\alpha)=\sum_{a} \int_{0}^{x_{a}^{n+1}} c_{a}(\omega) d \omega+\frac{1}{\theta} \sum_{w \in W} \sum_{k \in p_{w}} \hat{f}_{k(n+1)}^{w} \ln \hat{f}_{k(n+1)}^{w} \quad, \quad \text { in } \quad \text { which }
$$
$x_{a}^{n+1}=\sum_{w} \sum_{k} f_{k(n+1)}^{w} \delta_{a, k}^{w}$, the traffic flow of the user without ATIS is updated, $\hat{f}_{k(n+1)}^{w}=(1-\alpha) \hat{f}_{k(n)}^{w}+\alpha \hat{h}_{k(n)}^{w}$

Step4 : Convergence check。If it meets $\sqrt{\sum_{a}\left(x_{a}^{(n+1)}-x_{a}^{(n)}\right)^{2}} / \sum_{a} x_{a}^{(n)} \leq \varepsilon$, $\left.x_{a}^{(n)}=x_{a}^{1(n)}+x_{a}^{2(n)}\right)$, it stops, or $n=n+$, and it turns to step 2 to continue computation.

\section{2) Examples}

The following network example is used. If the travel time of each route section obeys normal distribution, the market share of ATIS is $30 \%$, and the reliability level is $80 \%$, the variance function of expected travel time and travel time of each route section on network is shown in the following table. And $\theta=1, q_{w}=100$.

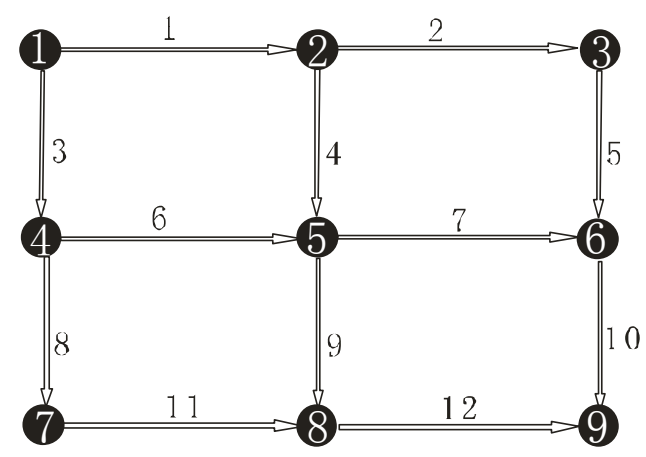

Figure 20. Example Network

Table 20. Impedance Function of Travel Time on Each Route Section

\begin{tabular}{|c|r|r|r|r|r|r|}
\hline $\begin{array}{c}\text { Number of route } \\
\text { section }\end{array}$ & 1 & 2 & 3 & 4 & 5 & 6 \\
\hline $\begin{array}{c}\text { Expected travel } \\
\text { time function }\end{array}$ & $\begin{array}{c}1 \\
0+\mathrm{v}\end{array}$ & $\begin{array}{c}5 \\
+3 \mathrm{v}\end{array}$ & $\begin{array}{c}2 \\
0+\mathrm{v}\end{array}$ & $\begin{array}{c}1 \\
0+2 \mathrm{v}\end{array}$ & $\begin{array}{r}1 \\
5+3 \mathrm{v}\end{array}$ & $\begin{array}{r}2 \\
0+\mathrm{v}\end{array}$ \\
\hline $\begin{array}{c}\text { Variance travel } \\
\text { time function }\end{array}$ & $v^{2}$ & $v$ & $v$ & $4 v$ & 16 & $25 v^{2}$ \\
\hline $\begin{array}{c}\text { Number of route } \\
\text { section }\end{array}$ & 7 & 8 & 9 & 1 & 1 & 1 \\
\hline
\end{tabular}




\begin{tabular}{|c|r|r|r|r|r|r|}
\hline $\begin{array}{c}\text { Expected travel } \\
\text { time function }\end{array}$ & $\begin{array}{r}1 \\
0+2 \mathrm{v}\end{array}$ & $+2 \mathrm{v}$ & $\begin{array}{r}2 \\
0+\mathrm{v}\end{array}$ & $\begin{array}{r}1 \\
0+\mathrm{v}\end{array}$ & $\begin{array}{r}5 \\
+3 \mathrm{v}\end{array}$ & $\begin{array}{r}5 \\
+3 \mathrm{v}\end{array}$ \\
\hline $\begin{array}{c}\text { Variance travel } \\
\text { time function }\end{array}$ & $9 v$ & 2 & $5 v^{2} v$ & $4 v$ & $v^{2}$ & $4 v^{2}$ \\
\hline
\end{tabular}

The example results are stored in the Pentium(R) with the memory of 256MB by using Matlab language. The results are shown in Table 21 and 22. The termination indicator of the algorithm is $\varepsilon=0.01$.

Table 21. Traffic Flow of Route Sections on Equilibrium

\begin{tabular}{|c|c|c|c|c|c|c|}
\hline $\begin{array}{l}\text { Num } \\
\text { ber of } \\
\text { routes }\end{array}$ & 1 & 2 & 3 & 4 & 5 & 6 \\
\hline $\begin{array}{l}\text { Balan } \\
\text { ced flow }\end{array}$ & $\begin{array}{c}63 . \\
0351 \\
\end{array}$ & $\begin{array}{c}12 . \\
0353\end{array}$ & $\begin{array}{r}36 . \\
967 \\
\end{array}$ & $\begin{array}{r}50 . \\
9463 \\
\end{array}$ & $\begin{array}{r}12 . \\
1232\end{array}$ & $\begin{array}{r}38 . \\
8971 \\
\end{array}$ \\
\hline $\begin{array}{l}\text { Num } \\
\text { ber of } \\
\text { routes }\end{array}$ & 7 & 8 & 9 & 10 & 11 & 12 \\
\hline $\begin{array}{l}\text { Balan } \\
\text { ced flow }\end{array}$ & $\begin{array}{c}10 . \\
3212\end{array}$ & $\begin{array}{l}3.2 \\
342\end{array}$ & $\begin{array}{r}73 . \\
1212\end{array}$ & $\begin{array}{c}23 . \\
567\end{array}$ & $\begin{array}{r}3.2 \\
231\end{array}$ & $\begin{array}{r}75 . \\
9002\end{array}$ \\
\hline
\end{tabular}

\section{Two-mode Transportation Equilibrium Assignment Model under the Influence of Traffic Information}

\subsection{Assumption Conditions}

There are two transportation modes, public transportation and private cars, on network at the same time, and the ATIS occupancy is different, which makes the transportation modes have many route selection criterions. The transportation mode to be selected by the traveler depends on the travel cost of the mode. If the traveler has cognitive error on the minimal travel cost of each transportation mode, and the error obeys joint normal distribution, the probability selection model based on polynomial can be achieved. Which mode to be selected by the traveler depends on the cognition of the user on the minimum optimal reliable travel time, or the cognition of the user with ATIS and without ATIS on the minimum optimal reliable travel time. As the proportion of two types of users can be set as $\rho$ and $\rho^{\prime}$, we can get the conclusion that the final minimal optimal reliable travel time of each mode is

$$
\mu_{w}^{i}=\rho \tilde{\pi}_{w}^{i}+\rho^{\prime} \hat{\pi}_{w}^{i}
$$

In the formula, $\tilde{\pi}_{w}^{i}$ and $\hat{\pi}_{w}^{i}$ are the cognitive value of the user with ATIS and without ATIS on the minimal optimal reliable travel time of the $i$ transportation mode.

It is the same to route selection. And the route selection model based on Logit and polynomial probability can be achieved.

If the transportation modes are interdependent, and the mixed network equilibrium of two-route selection criterions of two transportation modes are considered, we can get the following network equilibrium conditions 


$$
\left.\begin{array}{l}
q_{w}^{i}=p_{w}^{i} q_{w}(i=1,2) \\
\tilde{f}_{k}^{w, i}\left(c_{k}^{w, i}-\pi_{w}^{i}\right)=0(i=1,2) \\
c_{k}^{w, i}-\pi_{w}^{i} \geq 0(i=1,2) \\
\hat{f}_{k}^{w}=p_{k}^{w, i} q_{w}^{i}(i=1,2) \\
p_{w}^{i}=\frac{\exp \left(-\gamma \mu_{w}^{i}\right)}{\sum_{l} \exp \left(-\gamma \mu_{w}^{l}\right)}(i=1,2) \\
p_{k}^{w, i}=\frac{\exp \left(-\theta_{i} c_{k}^{w, i}\right)}{\sum_{l} \exp \left(-\theta_{i} c_{l}^{w, i}\right)}(i=1,2)
\end{array}\right\}
$$

$q_{w}^{i}$ means the traffic flow of the $\mathrm{i}$ transportation mode, $p_{w}^{i}$ means the probability that the $\mathrm{i}$ transportation mode is selected, $\tilde{f}_{k}^{w, i}$ means the traffic flow of the $\mathrm{i}$ transportation mode on route $\mathrm{k}$ with ATIS, $\hat{f}_{k}^{w, i}$ represents the traffic flow of the i transportation mode on route $\mathrm{k}$ without ATIS, $\pi^{i}$ means the minimal optimal reliable travel time of OD on the $\mathrm{i}$ transportation mode between $\mathrm{w}, p_{k}^{w, i}$ means the probability that the route $\mathrm{k}$ on network of the i transportation mode is selected. And $\mu^{i}$ means the minimal optimal reliable travel time of OD on the i transportation mode between $\mathrm{w}$, and the value is determined by the formula (28). $\gamma_{\text {and }} \theta$ mean the randomness of mode selection and route selection.

\subsection{Two-mode Traffic Network Equilibrium Assignment Model under the Influence of Traffic Information}

The two-mode traffic network equilibrium assignment model under the influence of traffic information can use the following equivalent mathematical programming model.

$$
\begin{gathered}
\min Z\left(\tilde{f}_{k}^{w, 1}, \hat{f}_{k}^{w, 1}, \tilde{f}_{k}^{w, 2} \hat{f}_{k}^{w, 2}, x_{a}^{1}, x_{a}^{2}, q_{w}^{2}\right) \\
=\sum_{a} \int_{0}^{x_{a}^{1}} c_{a}^{1}(\omega) d \omega+\frac{1}{\hat{\theta}_{1}} \sum_{w} \sum_{k \in p_{w}} \hat{f}_{k}^{w, 1} \ln \hat{f}_{k}^{w, 1}+\sum_{a} \int_{0}^{x_{a}^{1}} c_{a}^{2}(\omega) d \omega \\
+\frac{1}{\hat{\theta}_{2}} \sum_{w} \sum_{k \in p_{w}} \hat{f}_{k}^{w, 2} \ln \hat{f}_{k}^{w, 2}+\sum_{w} \int_{0}^{q_{w}^{2}}\left(\frac{1}{\gamma} \ln \frac{\omega}{q_{w}^{2}-\omega}\right) d \omega \\
\text { s.t. } \sum_{k \in \mathrm{I}} \tilde{f}_{k}^{w, 1}=\left(q_{w}-q_{w}^{2}\right) \rho ; \\
\sum_{k \in \mathrm{I}} \hat{f}_{k}^{w, 1}=\left(q_{w}-q_{w}^{2}\right) \rho \\
\tilde{f}_{k}^{w, 1} \geq 0 ; \\
\hat{f}_{k}^{w, 1} \geq 0 \\
x_{a}^{1}=\sum_{w} \sum_{k \in \mathrm{I}}\left(\tilde{f}_{k}^{w, 1}+\hat{f}_{k}^{w, 1}\right) \delta_{a, k}^{w, 1} ; \\
\sum_{k \in \Pi} \tilde{f}_{k}^{w, 2}=q_{w}^{2} \rho ;
\end{gathered}
$$




$$
\begin{array}{r}
\sum_{k \in \Pi} \hat{f}_{k}^{w, 2}=q_{w}^{2} \rho^{\prime} ; \\
\tilde{f}_{k}^{w, 2} \geq 0 ; \\
\hat{f}_{k}^{w, 2} \geq 0 ; \\
x_{a}^{2}=\sum_{w} \sum_{k \in \Pi}\left(\tilde{f}_{k}^{w, 2}+\hat{f}_{k}^{w, 2}\right) \delta_{a, k}^{w, 2}
\end{array}
$$

The formula (30b), (30c), (30g) and (30h) are flow conservation conditions, and the formula $(30 \mathrm{~d}),(30 \mathrm{e}),(30 \mathrm{i})$ and $(30 \mathrm{~g})$ are non-negativity conditions. The formula (22f) and (30k) are the relationship between route section and route flow.

Equivalence proof:

For any mathematical programming problem, any local minimum point or stagnation point meets the first-order condition. If the first-order condition of the model is equal to the above equilibrium conditions, it indicates that the user balance condition on any minimum point is successful, which proves equivalent relationship. The model is a minimal problem with constraints, and the generalized lagrangian function is

$$
\begin{aligned}
& L\left(\tilde{f}_{k}^{w, 1}, \hat{f}_{k}^{w, 1}, \tilde{f}_{k}^{w, 2}, \hat{f}_{k}^{w, 2}, q_{w}^{2}, \tilde{\pi}_{w}^{1}, \hat{\pi}_{w}^{1}, \tilde{\pi}_{w}^{2}, \hat{\pi}_{w}^{2}, \tilde{\lambda}^{1}, \hat{\lambda}^{1}, \tilde{\lambda}^{2}, \hat{\lambda}^{2}\right) \\
& =\sum_{a} \int_{0}^{x_{a}^{1}} c_{a}^{1}(\omega) d \omega+\frac{1}{\hat{\theta}_{1}} \sum_{w} \sum_{k \in p_{w}} \hat{f}_{k}^{w, 1} \ln \hat{f}_{k}^{w, 1}+\sum_{a} \int_{0}^{x_{a}^{1}} c_{a}^{2}(\omega) d \omega+\frac{1}{\hat{\theta}_{2}} \sum_{w} \sum_{k \in p_{w}} \hat{f}_{k}^{w, 2} \ln \hat{f}_{k}^{w, 2} \\
& \quad+\sum_{w} \int_{0}^{q_{w}^{2}}\left(\frac{1}{\gamma} \ln \frac{\omega}{q^{w, 2}-\omega}\right) d \omega+\tilde{\pi}_{w}^{1}\left[\left(q_{w}-q_{w}^{2}\right) \rho-\sum_{k \in \mathrm{I}} \tilde{f}_{k}^{w, 1}\right]+\hat{\pi}_{w}^{1}\left[\left(q_{w}-q_{w}^{2}\right) \rho-\sum_{k \in \mathrm{I}} \hat{f}_{k}^{w, 1}\right] \\
& -\tilde{\lambda}^{1} \tilde{f}_{k}^{w, 1}-\hat{\lambda}^{1} \hat{f}_{k}^{w, 1}+\tilde{\pi}_{w}^{2}\left(q_{w}^{2} \rho-\sum_{k \in \Pi} \tilde{f}_{k}^{w, 2}\right)+\hat{\pi}_{w}^{2}\left(q_{w}^{2} \rho-\sum_{k \in \Pi} \hat{f}_{k}^{w, 2}\right)-\tilde{\lambda}^{2} \tilde{f}_{k}^{w, 2}-\hat{\lambda}^{2} \hat{f}_{k}^{w, 2}
\end{aligned}
$$

$\tilde{\pi}_{w}^{1}, \hat{\pi}_{w}^{1}, \tilde{\pi}_{w}^{2}, \hat{\pi}_{w}^{2}$ are lagrangian functions corresponding to flow conservation constraints $(30 \mathrm{~b})(30 \mathrm{c})(30 \mathrm{~g})(30 \mathrm{~h}) . \tilde{\lambda}^{1}, \hat{\lambda}^{1}, \tilde{\lambda}^{2}, \hat{\lambda}^{2}$ are lagrangian functions of flow non-negativity corresponding to (30d) (30e) (30i) (30g). According to K-T condition, lagrangian functions must meet the following requirements on extreme value point.

$$
\begin{gathered}
\partial L / \partial q_{w}^{2}=0 ; \\
\partial L / \partial \tilde{f}_{k}^{w, 1}=0, \quad \tilde{\lambda}^{1} \tilde{f}_{k}^{w, 1}=0, \quad \tilde{\lambda}^{1} \geq 0 ; \\
\partial L / \partial \hat{f}_{k}^{w, 1}=0, \quad \hat{\lambda}^{1} \hat{f}_{k}^{w, 1}=0, \quad \hat{\lambda}^{1} \geq 0 ; \\
\partial L / \partial \tilde{f}_{k}^{w, 2}=0, \quad \tilde{\lambda}^{2} \tilde{f}_{k}^{w, 2}=0, \quad \tilde{\lambda}^{2} \geq 0 ; \\
\partial L / \partial \hat{f}_{k}^{w, 2}=0, \quad \hat{\lambda}^{2} \hat{f}_{k}^{w, 2}=0, \quad \hat{\lambda}^{2} \geq 0 ;
\end{gathered}
$$

For the user with ATIS using public transportation,

$$
\partial L / \partial \tilde{f}_{k}^{w, 1}=c_{k}^{w, 1}-\tilde{\pi}_{w}^{1}-\tilde{\lambda}^{1}=0
$$

As $\tilde{\lambda}^{1} \tilde{f}_{k}^{w, 1}=0$, if $\tilde{f}_{k}^{w, 1}>0, \tilde{\lambda}^{1}=0$.From the above formula, we can get $c_{k}^{w, 1}=\tilde{\pi}_{w}^{1}$. If $\tilde{\lambda}^{1}>0, \tilde{f}_{k}^{w, 1}=0$,and $c_{k}^{w, 1}=\tilde{\pi}_{w}^{1}+\tilde{\lambda}^{1}>\tilde{\pi}_{w}^{1}$. For the user with ATIS using public transportation mode, the traffic flow should meet determinant user balance condition. 
$\tilde{\pi}_{w}^{1}$ is the minimal optimal reliable travel time of the first kind of the user using public transportation mode of OD between $w$.

For the user without ATIS using public transportation,

$$
\partial L / \partial \hat{f}_{k}^{w, 1}==c_{k}^{w, 1}+\frac{1}{\hat{\theta}_{1}}\left(\ln \hat{f}_{k}^{w, 1}+1\right)-\hat{\pi}_{w}^{1}-\hat{\lambda}^{1}=0 ;
$$

If $\hat{f}_{k}^{w, 1}>0, \hat{\lambda}^{1}=0$, and the above formula can be written as

$$
c_{k}^{w, 1}+\frac{1}{\hat{\theta}_{1}}\left(\ln \hat{f}_{k}^{w, 1}+1\right)-\hat{\pi}_{w}^{1}=0 ;
$$

The formula is used to compute the route flow of the traveler without using ATIS, as follows.

$$
\hat{f}_{k}^{w, 1}=\exp \left[-\hat{\theta}_{1}\left(c_{k}^{w, 1}-\hat{\pi}_{w}^{1}\right)-1\right]=\exp \left[-\hat{\theta} c_{k}^{w, 1}\right] \exp \left[\hat{\theta} \hat{\pi}_{w}^{1}-1\right] ;
$$

From the conservation constraint $(30 \mathrm{~b})$, we can get $p_{k}^{w, 1}=\frac{\exp \left[-\theta c_{k}^{w, 1}\right]}{\sum_{l} \exp \left[-\theta c_{l}^{w, 1}\right]}$. It

indicates that the user without ATIS selecting public transportation mode obeys route selection mode of logitc to select route, which meets the user equilibrium condition of random user.

Similarly, it can prove that the user with ATIS and without ATIS can satisfy the determinant user equilibrium and the random user equilibrium condition.

$$
\begin{gathered}
\partial L / \partial q_{w}^{2}=\frac{1}{\gamma} \ln \frac{q_{w}^{2}}{q_{w}-q_{w}^{2}}-\tilde{\pi}_{w}^{1} \rho-\hat{\pi}_{w}^{1} \rho^{\prime}+\tilde{\pi}_{w}^{2} \rho+\hat{\pi}_{w}^{2} \rho^{\prime}=0 ; \\
\tilde{\pi}_{w}^{1} \rho+\hat{\pi}_{w}^{1} \rho^{\prime}=\mu_{w}^{1}, \quad \tilde{\pi}_{w}^{2} \rho+\hat{\pi}_{w}^{2} \rho^{\prime}=\mu_{w}^{2}, \text { and we can get } \\
p_{w}^{2}=\frac{\exp \left(-\gamma \mu_{w}^{2}\right)}{\sum_{l} \exp \left(-\gamma \mu_{w}^{l}\right)} ;
\end{gathered}
$$

The separation of transportation modes on network meets separation model based on logit. We can see that the model is equivalent to the equilibrium defined in the above chapter, and the model is an equivalent model.

2) Model solution algorithm

Step0: defining effective route set. (using the improve Dial algorithm such as algorithm 5.2.)

Step1: $\quad c_{a}^{1}(0), c_{a}^{2}(0)$ is used to compute effective route impedance, and all-or-none algorithm is used to compute the route flow $\tilde{f}_{k(1)}^{w, 1}$ and $\tilde{f}_{k(1)}^{w, 2}$ of the traveler with ATIS under two modes. And formula (36) is used to compute the traffic flow $\hat{f}_{k(1)}^{w, 1}$ and $\hat{f}_{k(1)}^{w, 2}$ of the traveler without ATIS, and $\mathrm{n}=1$;

Step2: $\quad f_{k(n)}^{w, 1}\left(f_{k(n)}^{w, 1}=\tilde{f}_{k(n)}^{w, 1}+\hat{f}_{k(n)}^{w, 1}\right) 、 f_{k(n)}^{w, 2}\left(f_{k(n)}^{w, 2}=\tilde{f}_{k(n)}^{w, 2}+\hat{f}_{k(n)}^{w, 2}\right)$ is used to compute the new route impedance. And all-or-none algorithm is used to compute the route flow $\tilde{h}_{k(n)}^{w, 1}$ and $\tilde{h}_{k(n)}^{w, 2}$ of the traveler with ATIS. And formula (36) is used to compute the traffic flow $\hat{h}_{k(n)}^{w, 1}$ and $\hat{h}_{k(n)}^{w, 2}$ of the traveler without ATIS.

Step3: computing step size. 
$3.10 \leq \tilde{\alpha} \leq 1$, and $\tilde{f}_{k(n+1)}^{w}=(1-\alpha) \tilde{f}_{k(n)}^{w}+\alpha \tilde{h}_{k(n)}^{w}$. The following problems are solved to determine step size, $\tilde{\alpha}=\tilde{\alpha}^{*} \min Z(\tilde{\alpha})=\sum_{a} \int_{0}^{x_{a}^{n+1}} c_{a}^{1}(\omega) d \omega+\frac{1}{\theta} \sum_{w \in W} \sum_{k \in p_{w}} \hat{h}_{k(n)}^{w, 1} \ln \hat{h}_{k(n)}^{w, 1}$, in which $x_{a}^{n+1}=\sum_{w} \sum_{k} f_{k(n+1)}^{w} \delta_{a, k}^{w}$, and the traffic flow of the user with ATIS on updating route is $\tilde{f}_{k(n+1)}^{w}=(1-\alpha) \tilde{f}_{k(n)}^{w}+\alpha \tilde{h}_{k(n)}^{w}$ 、

$3.20 \leq \hat{\alpha} \leq 1$, and $\hat{f}_{k(n+1)}^{w}=(1-\alpha) \hat{f}_{k(n)}^{w}+\alpha \hat{h}_{k(n)}^{w}$ is used to solve a series searching problems to determine step size, $\hat{\alpha}=\alpha^{*}$

$$
\min Z(\alpha)=\sum_{a} \int_{0}^{x_{a}^{n+1}} c_{a}(\omega) d \omega+\frac{1}{\theta} \sum_{w \in W} \sum_{k \in p_{w}} \hat{f}_{k(n+1)}^{w} \ln \hat{f}_{k(n+1)}^{w} \quad, \quad \text { in } \quad \text { which }
$$

$x_{a}^{n+1}=\sum_{w} \sum_{k} f_{k(n+1)}^{w} \delta_{a, k}^{w}$, the traffic flow of the user without ATIS on updated route is $\hat{f}_{k(n+1)}^{w}=(1-\alpha) \hat{f}_{k(n)}^{w}+\alpha \hat{h}_{k(n)}^{w}$

$3.30 \leq \tilde{\alpha}_{2} \leq 1$, and $\tilde{f}_{k(n+1)}^{w, 1}=\left(1-\tilde{\alpha}_{2}\right) \tilde{f}_{k(n)}^{w, 2}+\tilde{\alpha}_{1} \tilde{h}_{k(n)}^{w, 2}$ is used to solve a series searching problems to determine step size, $\hat{\alpha}=\alpha^{*}$

$$
\min Z\left(\tilde{\alpha}_{2}\right)=\sum_{a} \int_{0}^{x_{a}^{n+1}} c_{a}^{1}(\omega) d \omega+\frac{1}{\theta} \sum_{w \in W} \sum_{k \in p_{w}} \hat{h}_{k(n)}^{w, 2} \ln \hat{h}_{k(n)}^{w, 2} \quad, \quad \text { in } \quad \text { which }
$$
$x_{a}^{n+1}=\sum_{w} \sum_{k} f_{k(n+1)}^{w, 2} \delta_{a, k}^{w}$, the traffic flow of the user with ATIS on updated route is $\tilde{f}_{k(n+1)}^{w, 2}=\left(1-\tilde{\alpha}_{2}\right) \tilde{f}_{k(n)}^{w, 2}+\tilde{\alpha}_{2} \tilde{h}_{k(n)}^{w, 2}$.

$3.20 \leq \hat{\alpha}_{2} \leq 1$, and $\hat{f}_{k(n+1)}^{w, 2}=\left(1-\hat{\alpha}_{2}\right) \hat{f}_{k(n)}^{w, 2}+\hat{\alpha}_{2} \hat{h}_{k(n)}^{w, 2}$, is used to solve a series searching problems to determine step size, $\hat{\alpha}=\alpha^{*}$

$$
\min Z\left(\hat{\alpha}_{2}\right)=\sum_{a} \int_{0}^{x_{a}^{n+1}} c_{a}^{2}(\omega) d \omega+\frac{1}{\theta} \sum_{w \in W} \sum_{k \in p_{w}} \hat{f}_{k(n+1)}^{w, 2} \ln \hat{f}_{k(n+1)}^{w, 2} \quad, \quad \text { in } \quad \text { which }
$$
$x_{a}^{n+1}=\sum_{w} \sum_{k} f_{k(n+1)}^{w, 2} \delta_{a, k}^{w}$, and the traffic flow of the user without ATIS on updated route is $\hat{f}_{k(n+1)}^{w, 2}=\left(1-\hat{\alpha}_{2}\right) \hat{f}_{k(n)}^{w, 2}+\hat{\alpha}_{2} \hat{h}_{k(n)}^{w, 2}$

Step4 : Convergence check. If it meets $\sqrt{\sum_{a}\left(x_{a}^{(n+1)}-x_{a}^{(n)}\right)^{2}} / \sum_{a} x_{a}^{(n)} \leq \varepsilon$, $\left.x_{a}^{(n)}=x_{a}^{1(n)}+x_{a}^{2(n)}\right)$, it stops, or $n=n+$, and it turns to step 2 to continue computation.

\section{3) Examples}

The network example of formula (20) is used. If the travel time of each route section obeys normal distribution, and the reliability level is $80 \%$, the variance function of expected travel time and travel time of each route section on network is shown in the following table. The market share of ATIS is $\rho=0.3, \hat{\theta}_{1}={ }_{1}, \hat{\theta}_{2}={ }_{1}, \gamma={ }_{2}, q_{w}={ }_{100}$. 


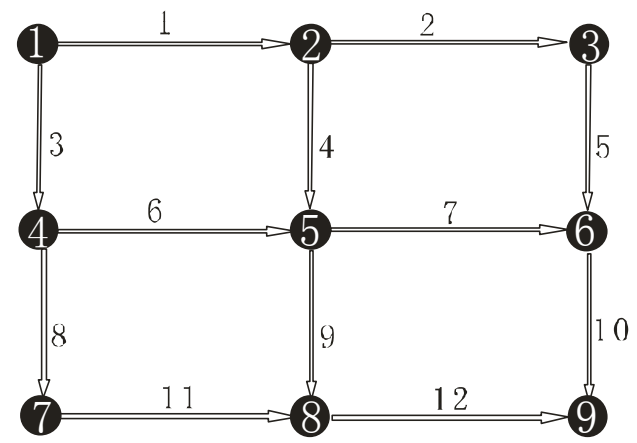

Figure 28. Example Network

The example results are stored in the Pentium(R) with the memory of $256 \mathrm{MB}$ by using Matlab language. The results are shown in Table 21 and 22. The termination indicator of the algorithm is $\varepsilon=0.01$.

\section{Table 31. Equilibrium Flow of Private Cars on Route Sections}

\subsection{Random Equilibrium Assignment Model of Two-mode Transportation Network under the Influence of Traffic Information}

1) Proposition of the model

$$
\begin{gathered}
\min Z\left(\tilde{f}_{k}^{w, 1}, \hat{f}_{k}^{w, 1}, \tilde{f}_{k}^{w, 2} \hat{f}_{k}^{w, 2}, x_{a}^{1}, x_{a}^{2}, q_{w}^{2}\right) \\
=\sum_{a} \int_{0}^{x_{a}^{1}} c_{a}^{1}(\omega) d \omega+\frac{1}{\hat{\theta}_{1}} \sum_{w} \sum_{k \in p_{w}} \hat{f}_{k}^{w, 1} \ln \hat{f}_{k}^{w, 1}+\frac{1}{\tilde{\theta}_{1}} \sum_{w} \sum_{k \in p_{w}} \tilde{f}_{k}^{w, 1} \ln \tilde{f}_{k}^{w, 1}+\sum_{a} \int_{0}^{x_{a}^{2}} c_{a}^{2}(\omega) d \omega \\
+\frac{1}{\hat{\theta}_{2}} \sum_{w} \sum_{k \in \Pi} \hat{f}_{k}^{w, 2} \ln \hat{f}_{k}^{w, 2}+\frac{1}{\tilde{\theta}_{2}} \sum_{w} \sum_{k \in p_{w}} \tilde{f}_{k}^{w, 2} \ln \tilde{f}_{k}^{w, 2}+\sum_{w} \int_{0}^{q_{w}^{2}}\left(\frac{1}{\gamma} \ln \frac{\omega}{q_{w}^{2}-\omega}\right) d \omega \\
\text { s.t. } \sum_{k \in \mathrm{I}} \tilde{f}_{k}^{w, 1}=\left(q_{w}-q_{w}^{2}\right) \rho ; \\
\sum_{k \in \mathrm{I}} \hat{f}_{k}^{w, 1}=\left(q_{w}-q_{w}^{2}\right) \rho \\
\tilde{f}_{k}^{w, 1} \geq 0 ; \\
\hat{f}_{k}^{w, 1} \geq 0 \\
\sum_{w} \sum_{k \in \mathrm{I}}\left(\tilde{f}_{k}^{w, 1}+\hat{f}_{k}^{w, 1}\right) \delta_{a, k}^{w, 1} ; \\
\sum_{k \in \Pi} \tilde{f}_{k}^{w, 2}=q_{w}^{2} \rho ; \\
\sum_{k \in \Pi} \hat{f}_{k}^{w, 2}=q_{w}^{2} \rho \\
\tilde{f}_{k}^{w, 2} \geq 0 ; \\
\hat{f}_{k}^{w, 2} \geq 0
\end{gathered} ;
$$

The formula (38b), (38c),$(38 \mathrm{~g})$ and $(38 \mathrm{~h})$ are flow conservation conditions, and the formula $(38 \mathrm{~d}),(38 \mathrm{e}),(38 \mathrm{i})$ and $(38 \mathrm{~g})$ are non-negativity conditions. The formula (38f) ) and (38k) are the relationship between route section and route flow.

Equivalence proof: 
For any mathematical programming problem, any local minimum point or stagnation point meets the first-order condition. If the first-order condition of the model is equal to the above equilibrium conditions, it indicates that the user balance condition on any minimum point is successful, which proves equivalent relationship. The model is a minimal problem with constraints, and the generalized lagrangian function is

$$
\begin{aligned}
& L\left(\tilde{f}_{k}^{w, 1}, \hat{f}_{k}^{w, 1}, \tilde{f}_{k}^{w, 2} \hat{f}_{k}^{w, 2}, q_{w}^{2}, \lambda_{1}^{1}, \tilde{\pi}_{w}^{1}, \hat{\pi}_{w}^{1}, \tilde{\pi}_{w}^{2}, \hat{\pi}_{w}^{2}, \tilde{\lambda}^{1}, \hat{\lambda}^{1}, \tilde{\lambda}^{2}, \hat{\lambda}^{2}\right) \\
& =\sum_{a} \int_{0}^{x_{a}^{1}} c_{a}^{1}(\omega) d \omega+\frac{1}{\hat{\theta}_{1}} \sum_{w} \sum_{k \in p_{w}} \hat{f}_{k}^{w, 1} \ln \hat{f}_{k}^{w, 1}+\frac{1}{\tilde{\theta}_{1}} \sum_{w} \sum_{k \in p_{w}} \tilde{f}_{k}^{w, 1} \ln \tilde{f}_{k}^{w, 1}+\sum_{a} \int_{0}^{x_{a}^{2}} c_{a}^{2}(\omega) d \omega \\
& +\frac{1}{\hat{\theta}_{2}} \sum_{w} \sum_{k \in \Pi} \hat{f}_{k}^{w, 2} \ln \hat{f}_{k}^{w, 2}+\frac{1}{\tilde{\theta}_{2}} \sum_{w} \sum_{k \in p_{w}} \tilde{f}_{k}^{w, 2} \ln \tilde{f}_{k}^{w, 2}+\sum_{w} \int_{0}^{q_{w}^{2}}\left(\frac{1}{\gamma} \ln \frac{\omega}{q_{w}^{2}-\omega}\right) d \omega \\
& +\tilde{\pi}_{w}^{1}\left[\left(q^{w}-q^{w, 2}\right) \square \rho-\sum_{k \in p_{w}} \tilde{f}_{k}^{w, 1}\right]+\hat{\pi}_{w}^{1}\left[\left(q^{w}-q^{w, 2}\right) \square \rho^{\prime}-\sum_{k \in p_{w}} \hat{f}_{k}^{w, 1}\right]-\tilde{\lambda}^{1} \tilde{f}_{k}^{w, 1}-\hat{\lambda}^{1} \hat{f}_{k}^{w, 1} \\
& \quad+\tilde{\pi}_{w}^{2}\left(q_{w}^{2} \rho-\sum_{k \in p_{w}} \tilde{f}_{k}^{w, 2}\right)+\hat{\pi}_{w}^{2}\left(q_{w}^{2} \rho-\sum_{k \in p_{w}} \hat{f}_{k}^{w, 2}\right)-\tilde{\lambda}^{2} \tilde{f}_{k}^{w, 2}-\hat{\lambda}^{2} \hat{f}_{k}^{w, 2} \\
& \tilde{\pi}_{w}^{1}, \quad \hat{\pi}_{w}^{1}, \quad \tilde{\pi}_{w}^{2}, \hat{\pi}_{w}^{2} \text { are lagrangian functions corresponding to flow conservation }
\end{aligned}
$$

constraints $(38 \mathrm{~b})(38 \mathrm{c})(38 \mathrm{~g})(38 \mathrm{~h}) . \tilde{\lambda}^{1}, \hat{\lambda}^{1}, \tilde{\lambda}^{2}, \hat{\lambda}^{2}$ are lagrangian functions of flow non-negativity corresponding to (38d) (38e) (38i) $(38 \mathrm{~g})$. According to K-T condition, lagrangian functions must meet the following requirements on extreme value point.

$$
\begin{array}{r}
\partial L / \partial q_{w}^{2}=0 \\
\partial L / \partial \tilde{f}_{k}^{w, 1}=0, \quad \tilde{\lambda}^{1} \tilde{f}_{k}^{w, 1}=0, \quad \tilde{\lambda}^{1} \geq 0 ; \\
\partial L / \partial \hat{f}_{k}^{w, 1}=0, \quad \hat{\lambda}^{1} \hat{f}_{k}^{w, 1}=0, \quad \hat{\lambda}^{1} \geq 0 ; \\
\partial L / \partial \tilde{f}_{k}^{w, 2}=0, \quad \tilde{\lambda}^{2} \tilde{f}_{k}^{w, 2}=0, \quad \tilde{\lambda}^{2} \geq 0 ; \\
\partial L / \partial \hat{f}_{k}^{w, 2}=0, \quad \hat{\lambda}^{2} \hat{f}_{k}^{w, 2}=0, \quad \hat{\lambda}^{2} \geq 0 ;
\end{array}
$$

For the user with ATIS using pubic transportation,

$$
\partial L / \partial \tilde{f}_{k}^{w, 1}=c_{k}^{w, 1}+\frac{1}{\tilde{\theta}_{1}}\left(\ln \tilde{f}_{k}^{w, 1}+1\right)-\tilde{\pi}_{w}^{1}-\tilde{\lambda}^{1}=0
$$

Obviously, $\tilde{f}_{k}^{w, 1}>0, \quad \tilde{\lambda}^{1}=0$. So the above formula can be written as

$$
c_{k}^{w, 1}+\frac{1}{\tilde{\theta}_{1}}\left(\ln \tilde{f}_{k}^{w, 1}+1\right)-\tilde{\pi}_{w}^{1}=0
$$

The above formula can be used to compute the route flow of the traveler without using ATIS, as follows.

$$
\tilde{f}_{k}^{w, 1}=\exp \left[-\tilde{\theta}_{1}\left(c_{k}^{w, 1}-\tilde{\pi}_{w}^{1}\right)-1\right]=\exp \left[-\tilde{\theta}_{1} c_{k}^{w, 1}\right] \exp \left[\tilde{\theta}_{1} \tilde{\pi}_{w}^{1}-1\right] ;
$$

From the flow conservation constraint (38d), we can get $\tilde{p}_{w}^{1}=\frac{\exp \left[-\tilde{\theta}_{1} c_{k}^{w, 1}\right]}{\sum_{l} \exp \left[-\tilde{\theta} c_{l}^{w, 1}\right]}$, which indicates that the user with ATIS selecting public transportation obey route selection mode of logit to select route, which meets the user equilibrium condition of random user. 
For the user without ATIS using pubic transportation,

$$
\partial L / \partial \hat{f}_{k}^{w, 1}=c_{k}^{w, 1}+\frac{1}{\hat{\theta}_{1}}\left(\ln \hat{f}_{k}^{w, 1}+1\right)-\hat{\pi}_{w}^{1}-\hat{\lambda}^{1}=0
$$

If $\hat{f}_{k}^{w, 1}>0, \quad \hat{\lambda}^{1}=0$ So the above formula can be written as

$$
c_{k}^{w, 1}+\frac{1}{\hat{\theta}_{1}}\left(\ln \tilde{f}_{k}^{w, 1}+1\right)-\hat{\pi}_{w}^{1}=0
$$

The above formula can be used to compute the route flow of the traveler without using ATIS, as follows.

$$
\hat{f}_{k}^{w, 1}=\exp \left[-\theta\left(c_{k}^{w, 1}-\hat{\pi}_{w}^{1}\right)-1\right]=\exp \left[-\hat{\theta}_{1} c_{k}^{w, 1}\right] \exp \left[\hat{\theta}_{1} \hat{\pi}_{w}^{1}-1\right]
$$

From the flow conservation constraint (38d), we can get $\tilde{p}_{w}^{1}=\frac{\exp \left[-\tilde{\theta}_{1} c_{k}^{w, 1}\right]}{\sum_{l} \exp \left[-\tilde{\theta} c_{l}^{w, 1}\right]}$,

which indicates that the user with ATIS selecting public transportation obey route selection mode of logit to select route, which meets the user equilibrium condition of random user.

Similarly, we can get that the user with ATIS and without ATIS selecting private cars meet the random user equilibrium condition. Due to the space limitation, it is not introduced here.

$$
\partial L / \partial q_{w}^{2}=\frac{1}{\gamma} \ln \frac{q_{w}^{2}}{q_{w}-q_{w}^{2}}-\tilde{\pi}_{w}^{1} \rho-\hat{\pi}_{w}^{1} \rho^{\prime}+\tilde{\pi}_{w}^{2} \rho+\hat{\pi}_{w}^{2} \rho^{\prime}=0
$$

As $\tilde{\pi}_{w}^{1} \rho+\hat{\pi}_{w}^{1} \rho^{\prime}=\mu_{w}^{1}, \tilde{\pi}_{w}^{2} \rho+\hat{\pi}_{w}^{2} \rho^{\prime}=\mu_{w}^{2} \quad$, we can get from the above formula that

$$
p_{w}^{2}=\frac{\exp \left(-\gamma \mu_{w}^{2}\right)}{\sum_{l} \exp \left(-\gamma \mu_{w}^{l}\right)}
$$

We can see that the separation of transportation modes on network also conforms to that based on logit. Above all, the model is equivalent to the equilibrium condition defined in the above chapter. And the model is an equivalent model.

2) Model solution algorithm

The mixed algorithm is used to solve the model in formula (38). The all-or-none flow load is only converted into random load on flow load of each step.

Step0: defining effective route set. (using the improve Dial algorithm such as algorithm 5.2.)

Step1: $\quad c_{a}^{1}(0), c_{a}^{2}(0)$ is used to compute effective route impedance, and all-or-none algorithm is used to compute the route flow $\tilde{f}_{k(1)}^{w, 1}$ and $\tilde{f}_{k(1)}^{w, 2}$ of the traveler with ATIS under two modes. And formula (36) is used to compute the traffic flow $\hat{f}_{k(1)}^{w, 1}$ and $\hat{f}_{k(1)}^{w, 2}$ of the traveler without ATIS, and $n=1$;

Step2: $\quad f_{k(n)}^{w, 1}\left(f_{k(n)}^{w, 1}=\tilde{f}_{k(n)}^{w, 1}+\hat{f}_{k(n)}^{w, 1}\right) 、 f_{k(n)}^{w, 2}\left(f_{k(n)}^{w, 2}=\tilde{f}_{k(n)}^{w, 2}+\hat{f}_{k(n)}^{w, 2}\right)$ is used to compute the new route impedance. And all-or-none algorithm is used to compute the route flow $\tilde{h}_{k(n)}^{w, 1}$ and $\tilde{h}_{k(n)}^{w, 2}$ of the traveler with ATIS. And formula (36) is used to compute the traffic flow $\hat{h}_{k(n)}^{w, 1}$ and $\hat{h}_{k(n)}^{w, 2}$ of the traveler without ATIS.

Step3: computing step size. 
$3.10 \leq \tilde{\alpha} \leq 1$, and $\tilde{f}_{k(n+1)}^{w}=(1-\alpha) \tilde{f}_{k(n)}^{w}+\alpha \tilde{h}_{k(n)}^{w}$. The following problems are solved to determine step size, $\tilde{\alpha}=\tilde{\alpha}^{*} \min Z(\tilde{\alpha})=\sum_{a} \int_{0}^{x_{a}^{n+1}} c_{a}^{1}(\omega) d \omega+\frac{1}{\theta} \sum_{w \in W} \sum_{k \in p_{w}} \hat{h}_{k(n)}^{w, 1} \ln \hat{h}_{k(n)}^{w, 1}$, in which $\quad x_{a}^{n+1}=\sum_{w} \sum_{k} f_{k(n+1)}^{w} \delta_{a, k}^{w}$, and the traffic flow of the user with ATIS on updating route is $\tilde{f}_{k(n+1)}^{w}=(1-\alpha) \tilde{f}_{k(n)}^{w}+\alpha \tilde{h}_{k(n)}^{w}$ 、

$3.20 \leq \hat{\alpha} \leq 1$, and $\hat{f}_{k(n+1)}^{w}=(1-\alpha) \hat{f}_{k(n)}^{w}+\alpha \hat{h}_{k(n)}^{w}$ is used to solve a series searching problems to determine step size, $\hat{\alpha}=\alpha^{*}$

$$
\min Z(\alpha)=\sum_{a} \int_{0}^{x_{a}^{n+1}} c_{a}(\omega) d \omega+\frac{1}{\theta} \sum_{w \in W} \sum_{k \in p_{w}} \hat{f}_{k(n+1)}^{w} \ln \hat{f}_{k(n+1)}^{w} \quad, \quad \text { in } \quad \text { which }
$$

$x_{a}^{n+1}=\sum_{w} \sum_{k} f_{k(n+1)}^{w} \delta_{a, k}^{w}$, the traffic flow of the user without ATIS on updated route is $\hat{f}_{k(n+1)}^{w}=(1-\alpha) \hat{f}_{k(n)}^{w}+\alpha \hat{h}_{k(n)}^{w}$

$3.30 \leq \tilde{\alpha}_{2} \leq 1$, and $\tilde{f}_{k(n+1)}^{w, 1}=\left(1-\tilde{\alpha}_{2}\right) \tilde{f}_{k(n)}^{w, 2}+\tilde{\alpha}_{1} \tilde{h}_{k(n)}^{w, 2}$ is used to solve a series searching problems to determine step size, $\hat{\alpha}=\alpha^{*}$

$$
\min Z\left(\tilde{\alpha}_{2}\right)=\sum_{a} \int_{0}^{x_{a}^{n+1}} c_{a}^{1}(\omega) d \omega+\frac{1}{\theta} \sum_{w \in W} \sum_{k \in p_{w}} \hat{h}_{k(n)}^{w, 2} \ln \hat{h}_{k(n)}^{w, 2} \quad, \quad \text { in } \quad \text { which }
$$
$x_{a}^{n+1}=\sum_{w} \sum_{k} f_{k(n+1)}^{w, 2} \delta_{a, k}^{w}$, the traffic flow of the user with ATIS on updated route is $\tilde{f}_{k(n+1)}^{w, 2}=\left(1-\tilde{\alpha}_{2}\right) \tilde{f}_{k(n)}^{w, 2}+\tilde{\alpha}_{2} \tilde{h}_{k(n)}^{w, 2}$ 、

Step4: Convergence check. If it meets $\sqrt{\sum_{a}\left(x_{a}^{(n+1)}-x_{a}^{(n)}\right)^{2}} / \sum_{a} x_{a}^{(n)} \leq \varepsilon$, $\left.x_{a}^{(n)}=x_{a}^{1(n)}+x_{a}^{2(n)}\right)$, it stops, or $n=n+$, and it turns to step 2 to continue computation.

\section{Conclusion}

The paper firstly analyzes the fact that many transportation modes coexist. Considering the equilibrium condition of network between transportation modes under interactive and not interactive situations, the paper establishes equivalent equilibrium assignment model and gives the relevant proof. Next, the paper divides the user on network into the user with advanced information system and the user without advanced information system. As the users with ATIS have advanced traffic information, so they can accurately know the information of network, and select the route with the minimal and reliable travel time. But the users without ATIS select the route with the minimal and reliable travel time by themselves. Generally speaking, the flow of the user is assigned on the network according to random law. The paper analyzes the multi-criterion equilibrium conditions, establishes equilibrium assignment model based on two criterions and gives the relevant proof. Lastly, the paper combines two modes and the equilibrium of two assignment criterions, establishes determinate and random equilibrium assignment model of two criterions and two modes, proposes the corresponding algorithm and gives the examples, which achieves the expected effect. 


\section{References}

[1] H. Lu, W. Wang and Lijiang, "Evaluation system of urban traffic management [M]", Beijing: China Communications Press, (2003)

[2] H. Liu, "Study on reliability of urban traffic network [D]", Southwest Jiaotong University, (2004).

[3] Y. Yu, "Analysis on feasibility of route network travel time based on statistics [D]", Beijing Jiaotong University, (2006), p. 5.

[4] X. Zhao, "Study and application of route system two-phase programming methods based on network reliability [D]", Zhengzhou University, (2006), p. 4.

[5] A. Liu and P. Yang, "Study on mixed traffic equilibrium assignment model and algorithm [D]", Highway Traffic Technology, vol. 3, no. 13, (1996), pp. 21-28.

[6] H. Huang, "Analysis and practice of urban traffic network equilibrium [M]", Beijing: China Communications Press, (1994).

[7] M. G. H. Bell and Y. lida, "Transportation network analysis [M]", John Wiley and Sons inc., (1997).

[8] Z. Ruan, and A. Luo, "Trust-region algorithm of traffic equilibrium assignment [J]", Statistics and Decision, vol. 10, (2011), pp. 34-36.

[9] X. Xia and B. Fan, "Multi-user road network equilibrium assignment model based on generalized cost [J]", Journal of Transportation and Highway Engineering, vol. 03, (2009), pp. 76-80.

\section{Author}

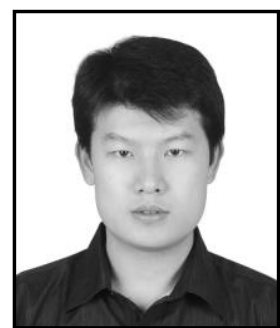

Liu Yansong, male, he was born in 1982. In 2006, he worked in System Integration Consultant, Jicheng Dianzi Ltd Co.2009 Working at Shandong Shandong management University (SDMU). He mainly studies Electronic Information Science and Technology, Intelligent traffic and Wireless sensor network etc. 
International Journal of Multimedia and Ubiquitous Engineering Vol.10, No.5 (2015) 